\title{
An analysis of COVID-19 and WHO global research roadmap: knowledge mapping and future research agenda
}

\author{
Mohammad Ashraful Mobin ${ }^{1,2}$ (D) Masnun Mahi ${ }^{3,1}$ (D) M. Kabir Hassan ${ }^{4}$ (D) \\ Marzia Habib $^{5}$ (D) Shabiha Akter ${ }^{6,1}$ (D) Tahmina Hassan $^{7}$
}

Received: 16 April 2020 / Revised: 28 July 2021 / Accepted: 8 September 2021 /

Published online: 9 November 2021

(c) Eurasia Business and Economics Society 2021

\begin{abstract}
In this study, we review the publications on the COVID-19 pandemic indexed in the Scopus database from 1st January to 15 th October 2020, using bibliometric analysis. In essence, we evaluate the publications against the global research roadmap outlined by the World Health Organization (WHO). We find that academic publications are contributed by researchers worldwide, with the USA and China being the top contributors in several parameters. We show that most publications are clustered around the issues related to the outbreak, diagnosis, candidate medicine, and disease treatment by analyzing publication keywords. Besides, considerable importance is given to unravel the connection between the present viral disease with its previous strains and the anxiety and stress-related problems that arise from the COVID-19. We further visualize the knowledge structure of the publication with thematic mapping and illustrate that mental health-related research denotes a specialized research theme besides public health issues being the basic theme. Our findings aid to comprehend how the scholarly publications have developed to address the prioritized research agendas by the WHO. Thus, our study can guide researchers to explore the less studied areas. Consequently, it will assist policymakers and governments in developing integrated and effective policies to fight the pandemic.
\end{abstract}

Keywords COVID-19 · Pandemic $\cdot$ Bibliometric $\cdot$ Knowledge mapping $\cdot$ Science mapping $\cdot$ World Health Organization

JEL Classification $\mathrm{H} 12 \cdot \mathrm{I} 10 \cdot \mathrm{I} 18 \cdot \mathrm{Y} 10$

M. Kabir Hassan

mhassan@uno.edu

Extended author information available on the last page of the article 


\section{Introduction}

Since the beginning of this new decade, the novel coronavirus, known as COVID19, has spread worldwide, first detected in Wuhan, China. On 30th January 2020, the World Health Organization (WHO) declared that the outbreak constitutes a Public Health Emergency of International Concern (PHEIC). The COVID-19 outbreak shocked the world and kept a challenge in front of the researchers to find a quick solution. Consequently, more than 400 participants worldwide joined the Global Research Forum of the WHO, including the member states representatives, health professionals, scientists, funders, and private sector representatives, to develop a proper strategy to control the COVID-19 pandemic. Taking the lessons from Ebola, SARS, Lassa fever, and Nipah, the forum felt the need for immediate and integrated research. As it was quoted in the WHO roadmap, "conducting research is linked to a moral obligation to learn as much as possible, as quickly as possible" (WHO, 2020 , p4). Research efforts are mandated to be integrated from the beginning, as the findings can help save lives when implemented appropriately. On 2nd March 2020, the scientific advisory group of the WHO R\&D blueprint met to assess the progress made since January 2020 and advise on additional prioritization of research activities. The cutting-edge priority areas ${ }^{1}$ identified by WHO include virus natural history, transmission and diagnostics, animal and environmental research on the virus origin, and management measures at the human-animal interface, epidemiological studies. Other priority areas include clinical management, infection prevention, and control, including health care workers' protection, candidate therapeutics $R \& D$, ethics considerations for research, and social sciences in the outbreak response.

Subsequently, the scientific arena has observed a rise in substantial research activities comprising diverse fields of study over the last few months. With the considerable research efforts made, it is crucial to evaluate the scientific outputs consistently to understand the existing strands of research, to identify the areas that are lacking required research, to find the essential future research avenue as well as to realign the research priority at both academic and policy levels. Evaluating scientific publications provides an intellectual structure and illustrates trends, theoretical evolution, and appearing or disappearing paradigms within public relations scholarship from an objective and quantitative perspective (Ki et al., 2019). Moreover, to improve the ongoing research, a collaborative research plan coupled with a coordinated and multi-disciplinary approach is needed. Accordingly, in this study, we conduct a bibliometric analysis of the available literature indexed Scopus database to evaluate the research outcomes. The study period essentially considers the publications that explicitly connote the roles of the WHO in their research from all available fields of study.

We use bibliometric analysis as the method allows us to analyze the written publications objectively (Ellegaard \& Wallin, 2015) and provide certain advantages to evaluate necessary information about a specific research area (Secinaro

\footnotetext{
1 A coordinated Global Research Roadmap by the WHO (Accessible at: https://www.who.int/blueprint/ priority-diseases/key-action/Roadmap-version-FINAL-for-WEB.pdf?ua=1).
} 
et al., 2020). With the significant expansion of the volume of scientific research, this method can efficiently track and monitor the research progress and present the state of the art of publications. Compared to other forms of literature review, bibliometric is a systematic, straightforward, and reproducible process that minimizes the intrinsic subjectivity of the conventional and systematic reviews (Della Corte et al., 2019). Bibliometric indicators, if properly analyzed, can give more consistency to the research project since they use statistics from different bibliographic databases (Campbell et al., 2010). Additional information could be gathered from quantitative methodologies that track advancements and take stock of rapidly developing literature. Correspondingly, the method helps organize bibliographic information in precise thematic fields (Merigó et al., 2015), which helps ascertain future research ( $\mathrm{Ji}$ et al., 2018). Besides, visualizing the bibliographic information through mapping allows scholars to understand research trends broadly and intuitively by highlighting the boundaries of the existing relevant intellectual territory and knowledge structure (Cobo et al., 2011).

Considering the benefits of bibliometric analysis in reviewing the literature, it has been popularly used in different subject areas. Incoherence, bibliometric analysis has been applied widely in medical research too, specifically in infectious disease and virology research (Azer, 2015; Hendrix, 2008; Liao et al., 2018). Subsequently, the method has been employed by some early-stage studies to evaluate the publications related to COVID-19 on different topics (Bonilla-Aldana et al., 2020; Danesh \& Ghavidel, 2020; El Mohadab et al., 2020; Gong et al., 2020; Homolak et al., 2020; Nowakowska et al., 2020). However, the existing bibliometric studies are conducted over a short span of time while the research on the COVID-19 pandemic is growing fast. The rapid growth of literature makes it difficult to comprehend the research concentration by the scholarly community. A literature review with their key attributes and their organized summary would help the research community and policymakers understand the development in research and integrate essential issues for future research. Hence, we make a humble attempt to address critical aspects of COVID-19 research by analyzing a relatively large bibliographic dataset and illustrating the findings precisely. In contrast to previous studies, we do not limit our analysis to identify the top contributing parties in the COVID-19 related studies; instead, we scientifically analyze and systematically highlight the research concentration through scientific mapping.

We factor in the WHO roadmap to evaluate research that has been conducted to understand the development of knowledge more evidently. The assessment has notable significance from the research as well as policy perspectives. Remarkably, the sheer abundance of research in this area can overwhelm decision-makers in the science-policy process, especially those new to the field. The failure to grasp the volume of available literature published on a sensitive issue like COVID-19 might lead to false recommendations and suggestions, hence suboptimal priorities in research and development agendas. In contrast, a clear picture of scientific research productivity in this field can assist relevant authorities (i.e., WHO, governments, etc.) to have a clear pathway about the research directions. Such guidance can be proved helpful in building in fighting the current and the future crises. 
Hence, through utilizing the bibliometric analysis method, we aim to answer the following essential research questions regarding the COVID-19 research-(1) Who are the most influential researchers and publication sources? (2) Which countries and institutions are leading the coronavirus research? (3) How are the country and institutional concentration of COVID-19 related research? How are they collaborating to advance knowledge? (4) What are the focused areas of research, underlying knowledge structure, and themes regarding the COVID-19 research so far? (5) Does the research cover all priority areas highlighted by the WHO global research roadmap? If not, what is missing?

To answer these major questions, we have reviewed 1,986 scientific publications retrieved from the Scopus database published between 1st January 2020 to 15th October 2020. Within the bounded sample, our study mainly discloses the most productive authors, journals, countries, and institutions as well as reveals their scholarly collaborative research efforts worldwide. Moreover, we analyze the most commonly used keywords by the researchers and scientifically illustrate the main research clusters and highlight the major themes around which the research has evolved to date.

Using the bibliographic data under analysis, we find that 9606 authors have contributed publications related to COVID-19 from a substantial number of institutions from several countries, and their work has been published in 1025 different publication outlets. While research covered numerous areas of study, most studies are related to the issues of medicine. We find that $\mathrm{Li}$ is the most productive author, having published 16 papers so far among all the contributing authors. However, Wang's name has come out as the most impactful researcher in terms of the total citation and h-index. Similarly, we find that the Lancet is the most publishing and the most influential publication outlet for the studies related to COVID-19 during this period. The USA has contributed the most so far by publishing 94 scientific documents, among which 23 are produced through the collaboration between the USA researchers and other country scholars. However, we find China's domination at the institutional level as five of their institutions list the top ten publishing institutions. While such findings provide a better picture of research adjustment of local needs and realities, our further analysis of country collaboration reveals that the researchers from the USA collaborated with countries their peers from the UK, Italy, Canada, and Germany mostly.

The findings from the perspectives of top authors, journals, countries, and collaboration are valuable information for the research community. Our publications and citation analysis assist researchers in drawing an overview of publishing activities in different disciplines related to the COVID-19 and understand changes in the impact of publications, scholars, institutions, and schools of thought of the fields and upon each other. Notably, the citation and equivalent indicator used in the analyses help us draw a literature map by identifying landmark studies and their growth. Furthermore, our analysis of keywords used by the authors in the published documents shows that 'covid-19', 'sars-cov-2', 'coronavirus', and 'pandemic' are mainly used at different frequencies. Then we examine the co-occurrence of keywords and illustrate the underlying research clusters using a two-dimensional plot, which reveals that most of the research that has been done is closely bunched together, focusing primarily on the outbreak, diagnosis, candidate medicine, and treatment. However, we 
find that a small but distinct group of researchers focused on the association between the present and the past outbreak of coronavirus-related disease. In contrast, other groups focus extensively on the problem related to 'anxiety' and 'stress' during the pandemic. Our visual mapping on the COVID-19 related publications provides insight into the developing path of existing concepts as well as a central concept's relationship with other ideas, or even a proposal of new concepts and new lineages that developed based on the existing ones in the field examined (i.e., thematic map).

Undeniably, the scientific community worldwide still has a lot to contribute to fighting the COVID-19 pandemic, as urged by the WHO. In view of that, our study discloses some outcomes from the perspective of academic thought that can guide scholars to observe underlying dynamics and help the academic community to identify the research gaps more adeptly. We believe the substantial description and visualization in our studies generated from the existing publications would help researchers further enrich the body of knowledge, hence make a notable contribution to the body of knowledge in this field. Unlike most bibliometric studies, we report the key indicators to find out the prominent authors, sources, countries, or institutions; instead, we provide crucial illustrations to understand the critical interrelatedness. Besides the academic contributions, our study holds some policy implications too. Notably, for the government, regulatory, and funding bodies, those who seek to promote research would cost-effectively look at the research productivity so far and take necessary measures to advance in the areas that require more attention.

The remainder of the paper is set out as follows: Sect. 2 explains the methodological aspects of the study by discussing the bibliometric analysis fundamentals and describing the sample. Section 3 presents and discusses the key bibliographic indicators to understand the scientific output in this research area. Section 4 concludes the study by summarizing the findings and suggesting future areas of research.

\section{Data and methodology}

To answer the research questions of this study, we utilize bibliographic information to evaluate published research on the COVID-19 pandemic. Our study employs several bibliometric indicators and science mapping techniques to analyze the published scientific documents on this issue. In this article, we use citation analysis to measure the impact of authors and publication sources. Citation analysis is the most conventional measure to approximate the scientific quality of individual researchers, universities, and institutions (Waltman et al., 2012) for evaluating the impact of publications (Frandsen \& Rousseau, 2005) measure of academic influence (Ellegaard $\&$ Wallin, 2015). If a publication or author's citation index is high, the publication or author is believed to impact the field (Feng et al., 2017). Besides, we make use of the $\mathrm{H}$-index to measure the impact of a published work. This indicator measures authors' productivity and influence in a particular and advantageous way to assess a scholar's research achievement in terms of quality and quantity (Hirsch, 2010; Hou et al., 2015). By definition, $H$-index indicates that the ' $h$ ' number of articles 
published by an author is cited at least ' $h$ ' times each. ${ }^{2}$ Hence, the metric helps to analyze the impact of scientific work or researcher by considering the total number of researches carried out (determined as the number of publications) and the consistency (determined as the number of citations in publications) on a single number. Besides, we analyze the keywords used in the research to understand the published work's focus and structure. This analysis uses literature keywords to build a semantic field map (Zupic \& Čater, 2015). It is a systematic method for scientifically discovering subfield linkages and tracking the phenomenon (Feng et al., 2017).

Furthermore, we consider the association of keywords through co-word analysis, a type of content analysis that extracts scientific maps of a field. This analysis helps researchers use a text's actual content directly to capture co-occurrence interactions in constructing the framework (Feng et al., 2017). Accordingly, we present the conceptual structure and thematic map of the existing research. For the conceptual structure analysis, we used Multiple Correspondence Analysis (MCA) that enabled us to identify the structure of existing research clusters through measuring the proximity of keywords used in the research (Demiroz \& Haase, 2019); as words are more similar in distribution, the closer they are represented in the map (Bieri, 2019). Besides, we use thematic mapping techniques that provide us with research topics, important expressions, and relationships. Four typologies of themes are defined according to the quadrant in which they are placed (Della Corte et al., 2019). The upper-right quadrant represents high centrality and density-the themes are developed and crucial for the research field. The upper-left quadrant signifies the highly developed but isolated themes or niche themes; themes have well developed internal links, however inconsequential external links. On the other hand, the lower-left quadrant presents the emerging or declining themes with low centrality and density, which are weakly developed and marginal. Finally, the lower-right quadrant is the quadrant of basic and transversal themes characterized by high centrality and low density. These themes are fundamental for a research field and concern general topics transversal to the different research areas of the field.

We retrieved data from the Scopus database to analyze the bibliographic data to answer the research questions posed earlier. Scopus database is the largest searchable citation and abstract source of searching literature expanded and updated incessantly. To create a representative set of scientific publication information, we specify the search string to get the publications that state the 'World Health Organization' in their publication explicitly from the available records in the database. The search string is as follows:

TITLE-ABS-KEY (("Corona Virus" AND "World Health Organization") OR ("Corona-Virus" AND "World Health Organization") OR ("COVID 19" AND "World Health Organization") OR ("COVID-19" AND "World Health Organization") OR ("ncov19" AND "World Health Organization") OR ("ncov 19" AND "World Health Organization") OR ("ncov-19" AND "World Health Organization") OR ("Novel Corona Virus" AND "World Health Organization") OR ("2019-nCoV" AND "World

\footnotetext{
${ }^{2}$ For instance, an H-index of 10 means that an author has published at least 10 papers (quantity) and that have each been cited at least 10 times (quality).
} 
Health Organization") OR ("2019 nCoV" AND "World Health Organization") OR ("Wuhan Virus" AND "World Health Organization") OR ("Wuhan-virus" AND "World Health Organization")).

The search includes a quest in title, abstract, and keywords of the published scientific documents from 1st January 2020 to 15 th October 2020. The period essentially experienced the rise of scientific publications since the outbreak of coronavirus on 31st December 2019. Using the search criteria specified, we retrieved bibliographic data for 2320 documents in the Scopus database. After looking at each record's title, abstract, and keywords, we eliminated the publications that were missing required bibliographic data fields (e.g., author name or affiliation, etc.). After doing the data cleaning, we obtained data of 1986 scientific documents for analysis. We analyze these documents' bibliographic data using the 'bibliometrix' package in R Language software developed by Aria and Cuccurullo (2017). The package essentially offers a wide array of options to process, analyze, and visualize data and help accurate publication data processing such as file conversion, term extraction, duplicate matching, and merging. At the same time, it offers researchers to analyze and present results with substantial descriptive analysis, matrix building, and network analysis (Aria \& Cuccurullo, 2017; Linnenluecke et al., 2019).

\section{Results and discussions}

This section presents the main bibliometric analysis results starting with the descriptive findings related to the vital information of the bibliographic data under analysis. Following that, we present our findings in different segments; mainly, we discuss our findings of an author analysis, publication sources analysis, the country, and institutional contributions analysis. Moreover, to comprehend the research frontiers regarding COVID-19 in line with the WHO roadmap, we analyze the research keywords and systematically present the significant research clusters and themes.

\subsection{Descriptive results}

The basic information of the data retrieved from the Scopus database during the period of the analysis is summarized in Table 1.

Table 1 shows that the published documents are mostly journal articles. It consists of around half $(51.76 \%)$ of all published documents under analysis, followed by a considerable review publication $\left(\mathrm{NP}^{3}=436\right)$. The scientific publications are contributed by a total of 9606 authors and published in 1,025 sources. Most of the works are collaborative, as the single-authored documents consist of only $12.64 \%$ of the total number of publications. The authors used 58,687 references and 3151 different keywords in the publications, and each document received 18 citations on average.

\footnotetext{
${ }^{3}$ NP: Number of Publications.
} 
Table 1 Main information about data

\begin{tabular}{|c|c|}
\hline \multicolumn{2}{|l|}{ Description } \\
\hline Documents & 1986 \\
\hline Sources (journals, books, etc.) & 1025 \\
\hline Authors & 9606 \\
\hline Authors of single-authored documents & 251 \\
\hline Authors of multi-authored documents & 9355 \\
\hline Average citations per documents & 17.63 \\
\hline References & 58,687 \\
\hline Author's keywords & 3151 \\
\hline \multicolumn{2}{|l|}{ Document types } \\
\hline Article & 1028 \\
\hline Article; data paper & 7 \\
\hline Editorial material & 155 \\
\hline Letter & 203 \\
\hline Review & 436 \\
\hline Conference paper & 11 \\
\hline Short survey & 23 \\
\hline Book chapter & 4 \\
\hline Erratum & 1 \\
\hline Note & 118 \\
\hline
\end{tabular}

Source: Authors' calculation

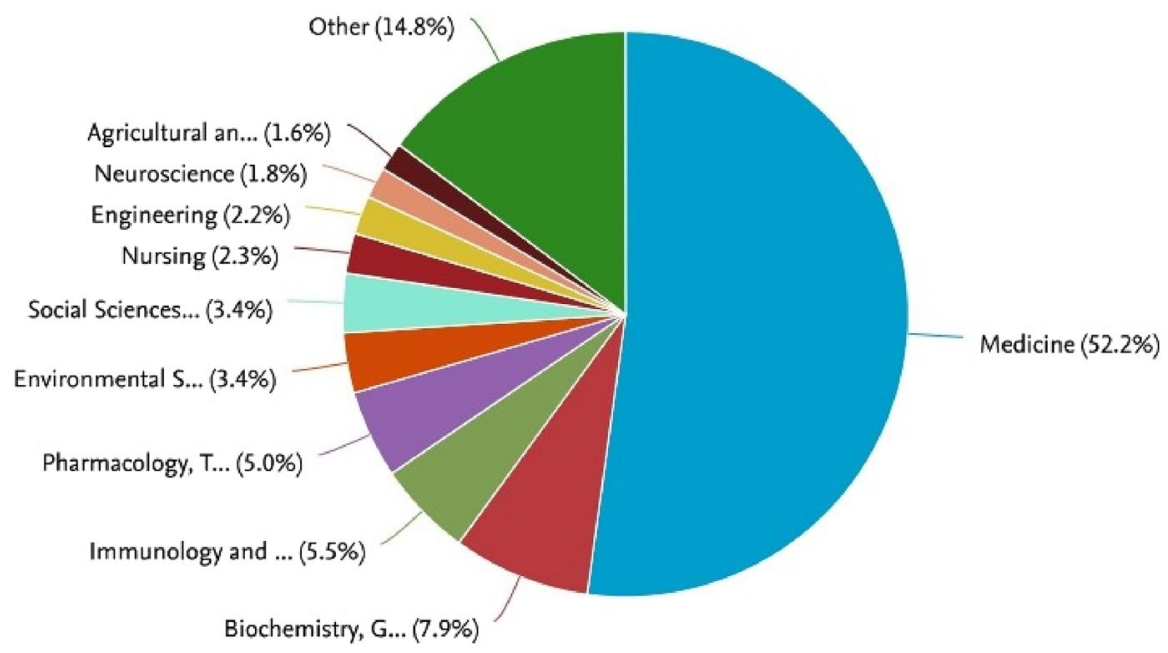

Fig. 1 Composition of publications by subject area. Source: Scopus database website illustration

Figure 1 shows the most common research areas categorized in the Scopus database in which the scientific documents on COVID-19 research are published. It comes as no surprise that most articles are placed in the research area of 'Medicine,' which consists of $52.20 \%$ of total published documents under consideration. 
Table 2 Most contributing authors

\begin{tabular}{|c|c|c|c|c|}
\hline Rank & Author & NP & $\mathrm{TC}$ & H-index \\
\hline 1 & $\mathrm{Li}$ & 16 & 173 & 6 \\
\hline 2 & Wang & 15 & 8045 & 8 \\
\hline 3 & Zhang & 13 & 205 & 5 \\
\hline 4 & $\mathrm{Li}$ & 12 & 7685 & 5 \\
\hline 5 & Liu & 12 & 629 & 5 \\
\hline 6 & Kumar & 9 & 31 & 3 \\
\hline 7 & Wang & 10 & 1766 & 7 \\
\hline 8 & Wang & 10 & 300 & 4 \\
\hline 9 & Dhama & 9 & 50 & 3 \\
\hline 10 & Rodriguez-Morales & 9 & 114 & 6 \\
\hline 11 & Sharma & 9 & 23 & 3 \\
\hline 12 & Wang & 9 & 7691 & 7 \\
\hline 13 & Gupta & 7 & 14 & 2 \\
\hline 14 & $\mathrm{Li}$ & 8 & 1069 & 5 \\
\hline 15 & $\mathrm{Li}$ & 7 & 1198 & 4 \\
\hline 16 & Liu & 8 & 191 & 5 \\
\hline 17 & Zhang & 8 & 25 & 2 \\
\hline 18 & Chen & 7 & 18 & 2 \\
\hline 19 & $\mathrm{Hu}$ & 7 & 7320 & 3 \\
\hline 20 & Khan & 6 & 53 & 3 \\
\hline
\end{tabular}

Source: Authors' calculation

$N P$ number of publications, $T C$ total citations

The research in 'Biochemistry, Genetics, and Molecular Biology,' 'Immunology and Microbiology,' and 'Pharmacology, Toxicology and Pharmaceutics' contribute significant portions in overall research outputs. Interestingly, we notice that a small but notable amount of research covers the issue of 'Environmental Science' and 'Social Science.'

Overall, the number of publications within a short period indicates that the issues related to COVID-19 cut much attention to the scholarly community, specially in response to the WHO research roadmap in diverse fields.

\subsection{Analysis of authors}

Table 2 presents the top twenty most influential authors published in Scopus indexed journals until now.

From the results presented in Table 2, we find that the top contributing authors' ranking varies considerably while considering different productivity and impact measures. By examining the number of publications, $\mathrm{Li}$ is the most productive author who published 16 scientific documents during this period. However, Li's works are not as impactful as the second most publishing author Wang considering the latter received 8,045 citations against the 15 published documents. Following 
Table 3 Most contributing publication sources

\begin{tabular}{llrrr}
\hline Rank & Source & NP & TC & H-index \\
\hline 1 & The Lancet & 35 & 8989 & 19 \\
2 & The BMJ & 32 & 385 & 8 \\
3 & International Journal of Environmental Research \& Public Health & 31 & 234 & 7 \\
4 & International Journal of Infectious Diseases & 22 & 482 & 7 \\
5 & International Journal of Research in Pharmaceutical Sciences & 19 & 4 & 1 \\
6 & Journal of Medical Virology & 19 & 731 & 11 \\
7 & Science of the Total Environment & 18 & 314 & 9 \\
8 & Journal of Medical Internet Research & 16 & 33 & 4 \\
9 & Travel Medicine and Infectious Disease & 16 & 164 & 5 \\
10 & International Journal of Surgery & 15 & 566 & 4 \\
11 & Journal of Travel Medicine & 14 & 873 & 7 \\
12 & Nature & 13 & 151 & 6 \\
13 & The Lancet Infectious Diseases & 13 & 1117 & 7 \\
14 & Dermatologic Therapy & 13 & 25 & 2 \\
15 & European Review for Medical and Pharmacological Sciences & 12 & 266 & 6 \\
16 & Journal of Pure and Applied Microbiology & 12 & 47 & 4 \\
17 & Public Health & 12 & 32 & 2 \\
18 & Chaos Solitons and Fractals & 12 & 43 & 3 \\
19 & International Journal of Pharmaceutical Research & 11 & 0 & 0 \\
20 & Journal of Infection and Public Health & 11 & 147 & 3 \\
\hline & Auth & &
\end{tabular}

Source: Authors' calculation

$N P$ number of publications, $T C$ total citations

Wang, Wang, $\mathrm{Li}$, and $\mathrm{Hu}$ are the most cited authors have a total citation (TC) of 7691, 7685, and 7320 citations. Similarly, Wang remains the most prominent author if the research impact is measured with $\mathrm{H}$-index. Wang and Wang are jointly ranked second since six of their publications received at least six citations among their published scientific documents. Even though Li's publications are not cited as much as the other author in the list, the author still ranked third jointly with RodriguezMorales having an $\mathrm{H}$-index value of 6.

\subsection{Analysis of publication sources}

Scholars must recognize the top journals of the related field. Identifying top journals helps the researchers decide which journals to choose to publish their scientific outputs and see the journals' overall focus in the research field. The exposure allows the researchers to communicate their findings quickly and efficiently to the scholarly community. Accordingly, we provide the top twenty publication outlets based on the publications listed in the Scopus databases that published studies related to coronavirus research in Table 3. 
Unlike the author rankings, the Lancet comes out as the most publishing and the most influential publication outlet considering the NP, TC, and $\mathrm{H}$-index value. The journal has published a total of 35 scientific documents listed in the Scopus database, given the search criterion specified, and all their publications received a total of 8989 citations during the period of analysis. Following the Lancet, the $\mathrm{BMJ}$ is the most publication source; however, this journal received significantly fewer citations (TC: 385) for the documents published than the former source. Instead, the articles published in the Lancet Infectious Diseases journal received 1117 citations in total. The numbers indicate the dominance of the Lancet journals in publishing scientific works in this area of research. Considering the citation analysis parameter, the Journal of Travel Medicine ranked third, having an aggregate citation of 873 .

On the other hand, the Journal of Medical Virology and Science of the Total Environment journals are the most impactful publication sources, following the Lancet considering H-index as the journals have the index value of 11 and 9, respectively. Overall, publication sources' analysis highlights that publishing more articles does not guarantee publications' quality since top publishing sources' ranking varies in the different benchmarks. Even some of the sources, such as the International Journal of Pharmaceutical Research, appeared as one of the top 20 publication outlets; however, the publications have not received any citation. Conversely, the Journal of Infection and Public Health has received 147 citations, more than other journals that published more scientific works. However, these metrics may not be used impartially to determine the quality of the journals; still, it provides valuable insights for the authors who will publish in the future in similar research areas.

Analysis of contributing countries and institutions

Information regarding the contributing country and institutions can be helpful to understand the geographic concentration of the research related to a particular area. Accordingly, we have presented the detailed country-specific publication information in Table 4 and institution-specific findings in Table 5.

As presented in Table 4, the research leader on COVID-19 is the USA (NP:94) up until now, followed by China (NP:73). India and Italy do not fall behind in publications, having published 67 and 53 scientific documents. Countries like Brazil, the UK, and Korea have ranked subsequently; however, the number of publications is significantly lesser than the top three countries on the list. To provide further insights on the nature of the country-specific publications, we make use of different indicators, namely Single Country Publications (SCP), Multiple Country Publications (MCP), and Multiple Country Publications Ratio (MCPR).

Researchers from all the countries in the top publishing country list have collaborated with other countries in different degrees except Singapore. The country is ranked 16th, contributing to 10 publications by its researchers, and all the authors are affiliated with their local institutions. Unsurprisingly, the USA and China occupy the first two positions in the list considering the total number of collaborative publications (MCP: 23) and (MCP: 20), followed by Italy (MCP: 11). However, the leading publishing countries that published more collaborative research against their SCP are Austria, Switzerland, and Saudi Arabia obtaining an MCPR value of $0.7778,0.5625$, and 0.5556 , correspondingly. To illustrate the country collaboration 
Table 4 Most contributing countries

\begin{tabular}{|c|c|c|c|c|c|}
\hline Rank & Country & NP & SCP & MCP & MCPR \\
\hline 1 & USA & 94 & 71 & 23 & 0.2447 \\
\hline 2 & China & 73 & 53 & 20 & 0.274 \\
\hline 3 & India & 67 & 61 & 6 & 0.0896 \\
\hline 4 & Italy & 53 & 42 & 11 & 0.2075 \\
\hline 5 & Brazil & 28 & 25 & 3 & 0.1071 \\
\hline 6 & UK & 26 & 18 & 8 & 0.3077 \\
\hline 7 & Korea & 22 & 20 & 2 & 0.0909 \\
\hline 8 & Canada & 18 & 12 & 6 & 0.3333 \\
\hline 9 & Germany & 18 & 14 & 4 & 0.2222 \\
\hline 10 & France & 17 & 13 & 4 & 0.2353 \\
\hline 11 & Switzerland & 16 & 7 & 9 & 0.5625 \\
\hline 12 & Spain & 15 & 10 & 5 & 0.3333 \\
\hline 13 & Iran & 14 & 9 & 5 & 0.3571 \\
\hline 14 & Japan & 11 & 7 & 4 & 0.3636 \\
\hline 15 & Mexico & 10 & 5 & 5 & 0.5 \\
\hline 16 & Singapore & 10 & 10 & 0 & 0 \\
\hline 17 & Austria & 9 & 2 & 7 & 0.7778 \\
\hline 18 & Hong Kong & 9 & 5 & 4 & 0.4444 \\
\hline 19 & Saudi Arabia & 9 & 4 & 5 & 0.5556 \\
\hline 20 & Malaysia & 7 & 6 & 1 & 0.1429 \\
\hline
\end{tabular}

Source: Authors' calculation

For a particular country, the MCPR ratio is calculated as $\mathrm{MCPR}=$ total number of multiple country publications/total publications

$N P$ number of publications, $S C P$ single country publications, $M C P$ multiple country publications, $M C P R$ multiple country publications ratio

further, we present the Country Collaboration World Map in Fig. 2. The blue color on the map indicates the existence of publications for a particular country on the issues under analysis, and the color grey indicates no publication. Besides, the countries with darker blue color represent more publishing countries while the red lines indicate the publishing countries' collaboration networks.

The countries that were collaborating most actively are the USA and the UK. Being the most publishing country, the USA has prominent scientific collaboration with China, Italy, Canada, Germany, and India. On the other hand, Chinese researchers collaborated with the UK and Italy researchers the most. Perhaps, the Chinese collaborations are strong with European countries as Europe became the epicenter of COVID-19, shifting from China. Hence, the collaborative publication information point towards the flow of knowledge from one country to another during the pandemic. On the contrary, Indian collaborative publications are primarily with Saudi Arabia and Italy besides China. While the countries show different degrees of collaboration in expanding and disseminating new knowledge, our analysis could 


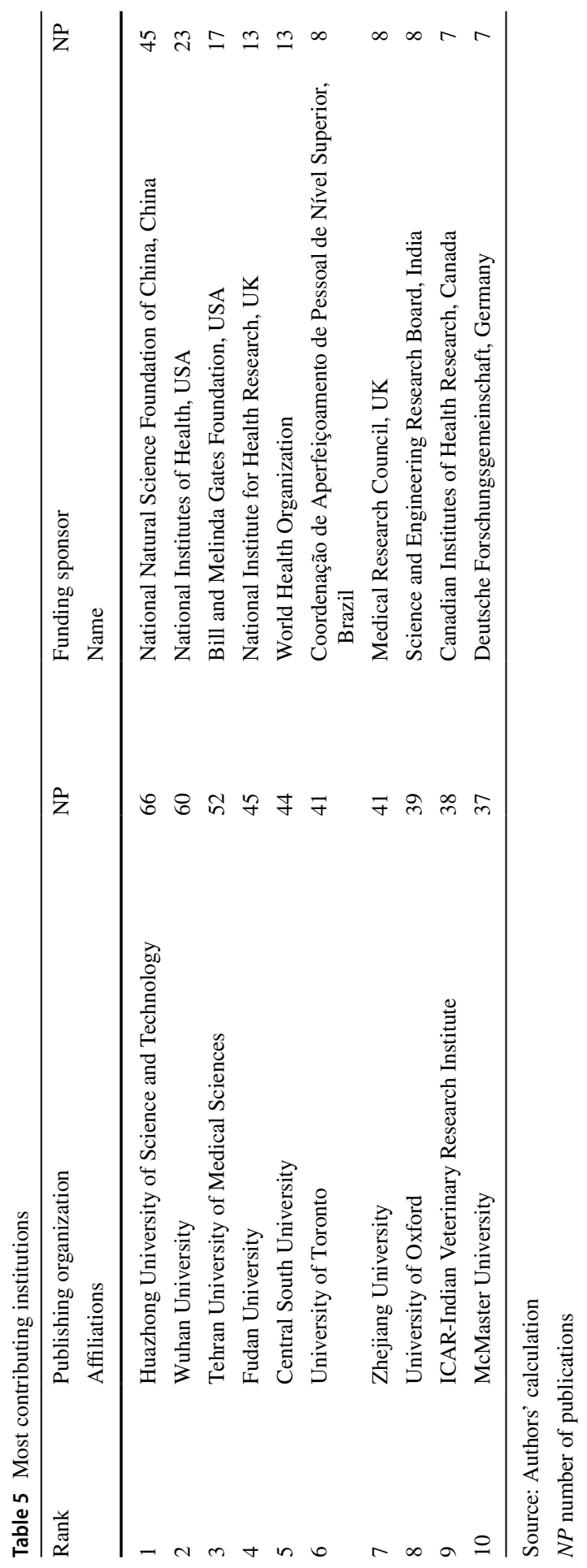




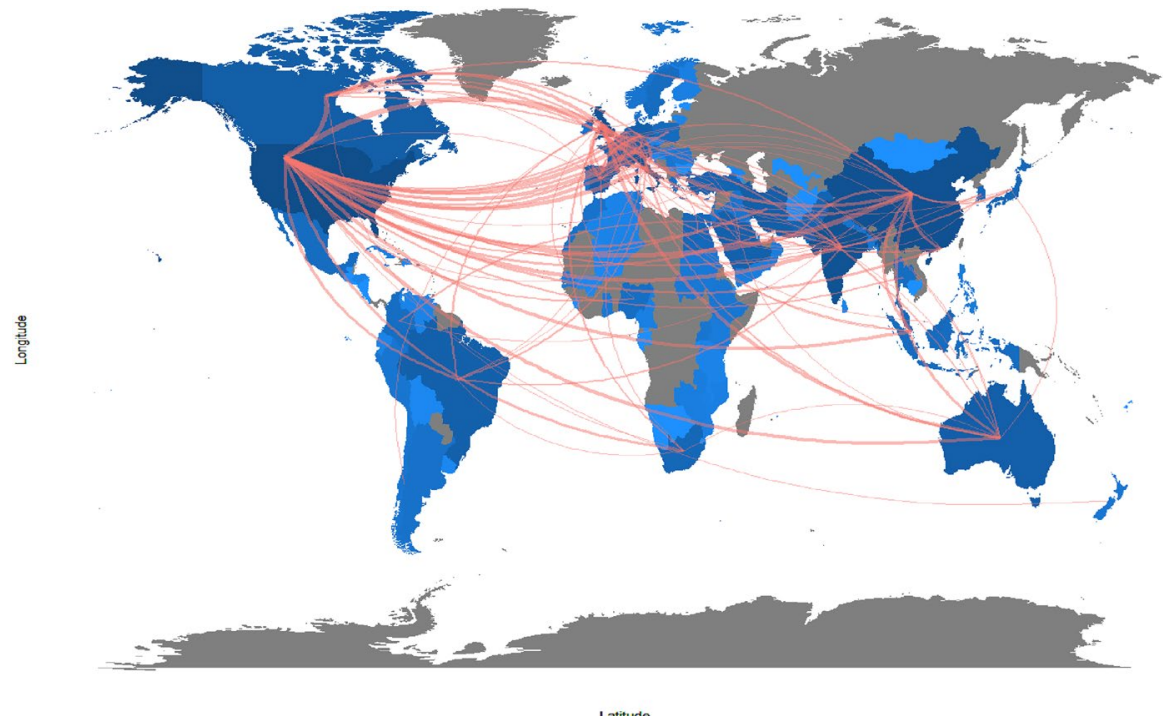

Fig. 2 Country collaboration world map. Source: Authors' illustration

not capture such activities by some countries; notable examples include Russia and some African nations.

Besides presenting the scientific publications at the country or macro level, we analyze the information at the micro or institution level too. Table 5 shows the contributions to scientific research of different organizations from two perspectives-the most contributing institutions in publications and the most supporting government and public organizations supporting the scientific works with required funding.

From the total number of publications perspective, we notice Chinese universities' dominance as Huazhong University of Science and Technology and Wuhan University occupy the top two positions, having contributed to 66 and 60 publications, respectively. The other Chinese universities on the list are Fudan University, Central South University, and Zhejiang University. Among the others, we find that two top-ranked research institutions are from Canada, namely, the University of Toronto and McMaster University, while other top-ranked universities belong to Iran, the UK, and India.

Unlike the top publishing institutions, funding sponsorship is not dominated by Chinese organizations extensively. However, the National Natural Science Foundation of China contributed to research and publications of 45 scientific documents, which is significantly higher than the second-ranked National Institutes of Health of the USA, which funded in 23 publications. Moreover, the third-placed funding sponsor is based in the USA - Bill and Melinda Gates Foundation. Also, the National Institute for Health Research of the UK and the WHO has sponsored scientific publications somewhat higher than the comparable other funding sponsors on the list. 
Table 6 Most frequently used author keywords

\begin{tabular}{llrrllll}
\hline Rank & Keywords & Freq. & $\%$ & Rank & Keywords & Freq. & $\%$ \\
\hline 1 & Covid-19 & 1016 & 43 & 11 & Pandemics & 31 & 1 \\
2 & Sars-cov-2 & 336 & 14 & 12 & Mental health & 28 & 1 \\
3 & Coronavirus & 309 & 13 & 13 & Diagnosis & 27 & 1 \\
4 & Pandemic & 200 & 8 & 14 & Outbreak & 26 & 1 \\
5 & 2019-ncov & 54 & 2 & 15 & Sars & 25 & 1 \\
6 & Public health & 54 & 2 & 16 & Prevention & 23 & 1 \\
7 & Novel coronavirus & 44 & 2 & 17 & Corona virus & 22 & 1 \\
8 & Epidemiology & 41 & 2 & 18 & Epidemic & 21 & 1 \\
9 & World Health Organization & 35 & 1 & 19 & Mortality & 21 & 1 \\
10 & Pneumonia & 33 & 1 & 20 & Pregnancy & 21 & 1 \\
\hline
\end{tabular}

Source: Authors' calculation

\subsection{Analysis of research keywords, knowledge structure, and major themes}

As discussed in the earlier sections, a substantial amount of scientific works has been published by the scholarly community worldwide. This section analyzes the keywords used in the published studies to comprehend the leading research frontiers. To do so, we present the most used keywords and illustrate the conceptual structure and themes of research through the co-occurrence of those keywords.

As shown in Table 1, the authors used a total of 3,151 different keywords in the relevant 1,986 scientific documents that we are analyzing. Among the most used keywords, the top twenty keywords based on their frequency of occurrences and the relative share of each keyword to total keywords (in percentage) are listed in Table 6.

With no surprise, most of the authors listed 'covid-19' as the main keywords, along with 'sars-cov-2' and 'coronavirus.' The first keywords appeared 1016 times in the published documents, consists $43 \%$ of the total keywords published in scholarly works during the period. On the other hand, the other two mostly used keywords that account for $14 \%$ and $13 \%$ of all the keywords used. However, we find the comparable keywords '2019-ncov' or 'novel coronavirus' are less frequently used by the researchers than the other keywords to refer to the disease. Each of the words consists of around $2 \%$ of the words author keywords. Instead, the pandemic issues cut a substantial amount of research interest and appeared 200 times or $8 \%$ of the total. Although we did not specify the keyword 'pandemic' in our search string, the notable appearance of keywords indicates the close link in research focused on different aspects of COVID-19.

We then conducted the Multiple Correspondence Analysis (MCA) of the keywords included in our bibliographic dataset. The analysis draws a conceptual structure of the field and K-means clustering to identify clusters of documents that express common concepts (Aria \& Cuccurullo, 2017). The conceptual structure of the keywords associated with the scientific publications on the topic of our interest is presented in Fig. 3. 


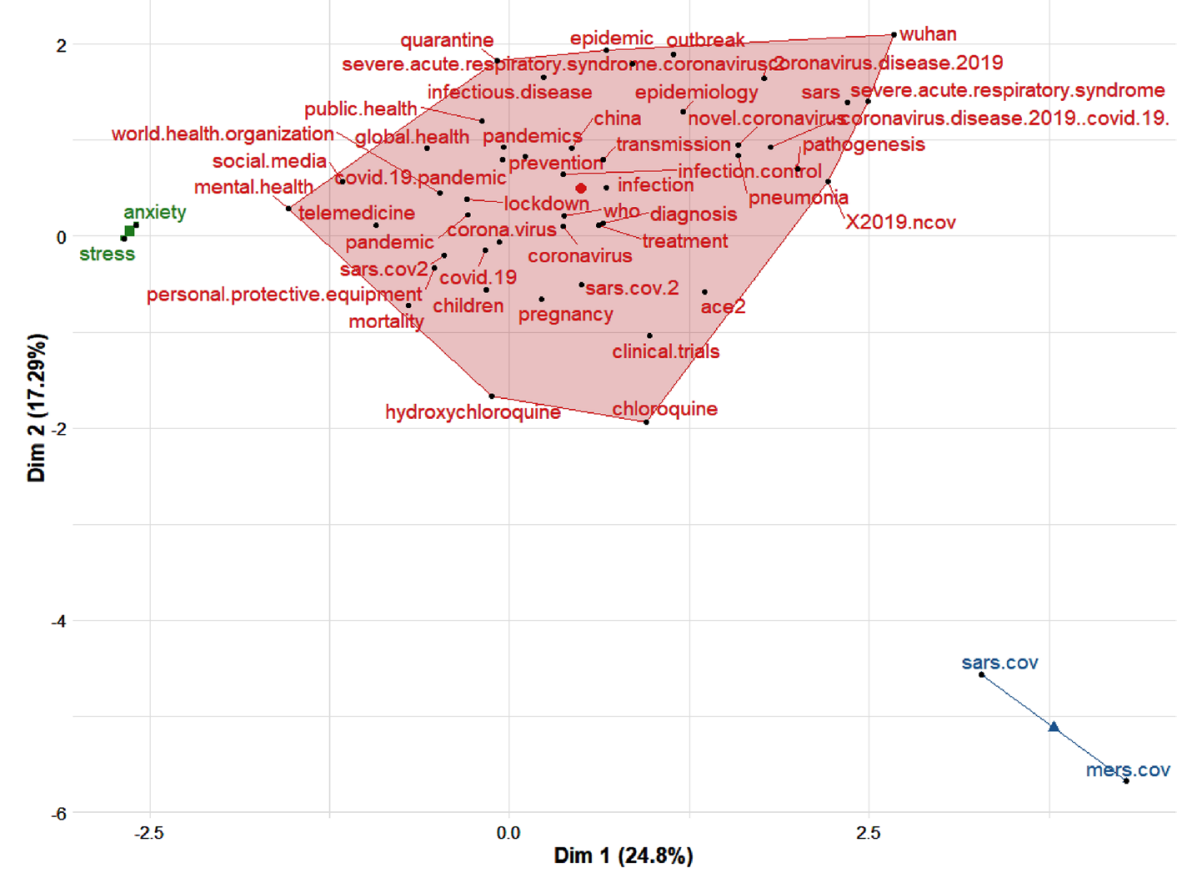

Fig. 3 Conceptual structure of research. Source: Authors' illustration

The illustration indicates that the published documents under our analysis can be organized into four primary clusters representing the intellectual structure of COVID-19 related studies. A detailed review of contents associated with each group is beyond the scope of the current study; however, we demonstrate the intellectual focus of the research undertaken based on their proximity or cluster. The most extensive research cluster is highlighted in red in the conceptual structure map. Such a large cluster indicates that most of the research is closely associated with each other at different degrees. A total of 48 keywords are associate with the red cluster, which covers from initial outbreak, pathogenesis, development of the health crisis to a global pandemic, containment measures such as quarantine, and the like. Also, the associated studies focus on epidemiology, diagnosis, and treatment to a significant extent. On the treatment part, we can notice research concentration on the candidate vaccines' clinical trials simultaneously using different medicine such as chloroquine and hydroxychloroquine. Inline, we notice a particular geographic focus of studies that are related to China or Wuhan. However, we do not find the distinctive presence of other countries or regions by analyzing the research cluster. Besides, the research related to COVID-19 has provided a particular focus on the impact on women and children, which is identifiable with keywords like 'pregnancy' or 'children.' Moreover, some studies have focused on the technological aspects like social media and telemedicine that represent a novel yet intently associated with the health crisis brought by COVID-19. 


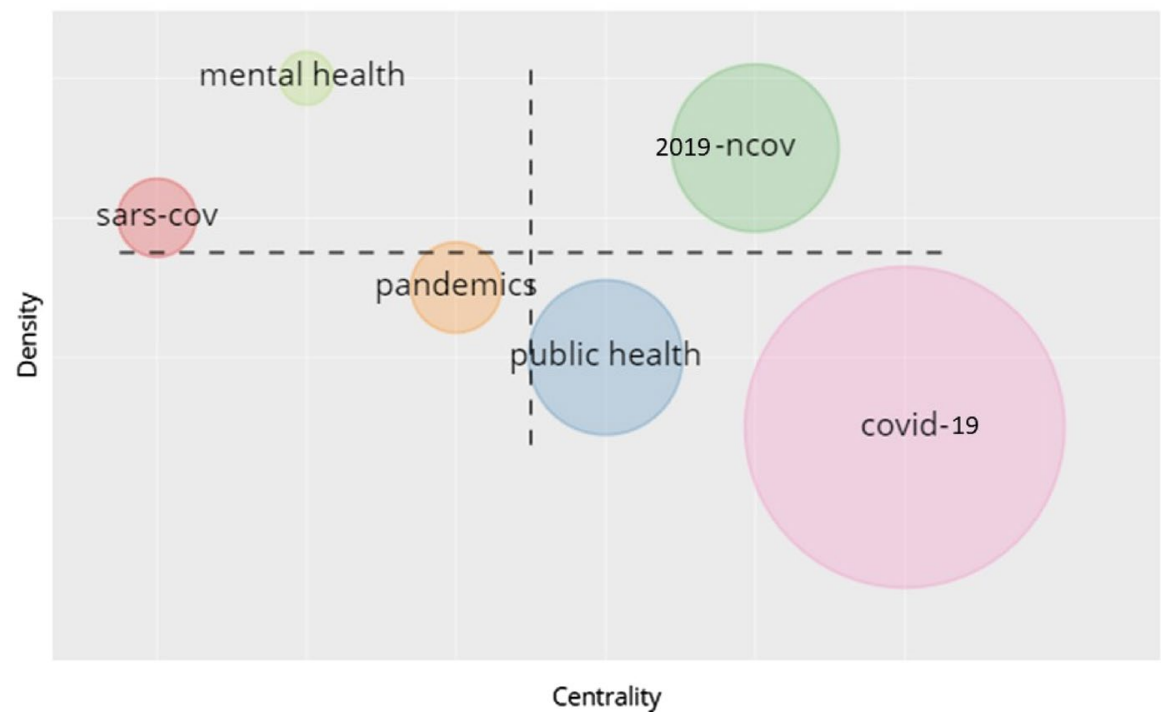

Fig. 4 Thematic mapping. Source: Authors' illustration (color figure online)

Although we notice the close association of research on mental health besides the physical health-related topics in the first cluster, we find that anxiety and stress have become unique research issues during this period. Accordingly, we see a small but distinct research cluster that focuses on these two issues as labeled by the green in the diagram. The COVID-19 pandemic causes physical health concerns and results in several psychological disorders (Salari et al., 2020). Besides, we find that the group of published documents investigating the linkage between the novel coronavirus and the earlier coronavirus outbreak represents a separate research cluster labeled in blue in the illustration. Specifically, the publications examine the linkages between 'mers cov' (Middle East respiratory syndrome coronavirus) and 'sars cov' (Severe acute respiratory syndrome-related coronavirus). Such research efforts are comprehensible since previous outbreaks experiences with similar viral agents have increased the capacity to contain these recurrent disease containment and control.

We further illustrate the knowledge structure of published research to date through a thematic map in Fig. 4. Compared to conceptual structure analysis, one distinguishable benefits of thematic mapping are that it helps distinguish the research concentration in different categories depending on the levels. The degree of the relation among various topics is termed as the centrality of a theme, while density indicates the advancement of a particular theme (Esfahani et al., 2019).

From Fig. 4, we notice two main research clusters directly related to 'covid-19' (color: pink) or '2019-ncov' (color: green). However, the themes are located in different quadrants based on their degree of concentration and association with related topics. The scientific publications come together under the theme titled '2019-ncov' presents the motor theme or the well-developed theme of research that mainly focused on the nature of the disease like pneumonia. The focus on the generic nature 
of the disease makes this a vital theme of the research in the existing stream of publications. On the other hand, the 'covid-19' represents the basic and transversal theme, consisting of the general topics transverse to the different research areas. The major keywords associated with the publications under this theme include transmission, prevention, diagnosis, and treatment, among others. Similar to the 'covid-19' theme, the map shows that 'public health' (color: blue) related research issues are also the general topics of interest associated with the other research areas and mainly concentrated on epidemiology and outbreak. Besides, we find research related to 'mental health' (color: olive green) along with 'sars-cov' (color: red) represents the specialized themes of research while studies on 'pandemic' (color: brown) characterize the emerging themes of research for the scientific publications under analysis.

\section{Conclusion and future research directions}

The academic community has carried out a remarkable amount of research since the outbreak of the COVID-19 pandemic. The WHO emphasizes scholarly contributions from different fields of study to deliver integrated knowledge and implement it appropriately to save lives. Inline, it is essential to evaluate the scholarly performances systematically to realize what has been done and what needs to be done. Consequently, we attempt to review the published literature in the light of the research roadmap by WHO. We utilize the bibliometric analysis to answer these vital issues. Our study reviews the literature in quantitative terms using the bibliographic data of 1986 publications listed in the Scopus database and identifies the top contributing authors, publication sources, countries, and institutions. In addition, we analyze and visualize their collaborative research efforts using a comprehensive science mapping $\mathrm{R}$ language package called bibliometrix. The summary of the major findings are presented against the respective research questions below:

Who are the most influential researchers and publication sources?

From our analysis, among the 9606 contributing authors in different fields of study, $\mathrm{Li}$ is the most productive author with total publications of 16 scientific documents. At the same time, Wang appears as the most impactful author in terms of total citations received and h-index. However, as a publication source, the Lancet comes out as the most publishing and the most impactful journal. It publishes 35 scientific works during the analysis period and has amassed 8989 citations in total for its published documents. Accordingly, the journal has received the highest $\mathrm{H}$-index value of 19 , which is significantly higher than the other top sources like the Journal of Medical Virology, Science of the Total Environment, or the BMJ. Also, we present that the publications are primarily concentrated in medicine $(52.2 \%)$. At the same time, 'Biochemistry, Genetics, and Molecular Biology', 'Immunology and Microbiology' and 'Pharmacology, Toxicology, and Pharmaceutics' are the other most researched areas.

Which countries and institutions are leading the coronavirus research? How are the country and institutional concentration of COVID-19 related research? How are they collaborating to advance knowledge? 
In our analysis of the geographic distribution of scientific studies and collaborative research outputs, we find that the USA is the country whose researchers have published more in terms of the total number of publications (NP: 94) and multicountry publications (MCP: 23). However, we find that countries like Austria, Switzerland, and Saudi Arabia are more active in collaborative publications compared to their country-specific scientific productivity. On the contrary, documents affiliated with Chinese universities dominated the total number of publications at the organizational level. Five out of ten top listed universities are from China, among which Huazhong University of Science and Technology tops the list. Also, universities or research institutions from Canada, Iran, the UK, and India are among the most actively publishing institutions.

What are the focused areas of research, underlying knowledge structure, and themes regarding the COVID-19 research so far?

Likewise, by using the science mapping technique, we visualize and trace the knowledge concentration of the field as well as distinguish the development of research themes. Before plotting the maps, we identify the most frequently used keywords and found that 'covid-19', 'sars-cov-2', 'coronavirus,' and 'pandemic' are used the most by authors to categorize their research.

Besides, the co-word analysis shows that the keywords are clustered closely in a single large research cluster. However, our analysis distinguishes the development of two other small but distinct research clusters that specially focused on the linkages between the different coronavirus (i.e., sars-cov \& mers-cov) alongside 'stress' and 'anxiety' related concerns during the pandemic. We further investigate the development of research themes and uncover that research directly related to 'covid-19', '2019-ncov', or 'public health' represents the basic and motor research themes. However, studies related to mental health problems constitute a specialized research area, while pandemics studies' interest represents an emerging research theme.

Does the research cover all priority areas highlighted by the WHO global research roadmap? If not, what is missing?

Predictably, the existing publications are dominated by medicine and other science-related areas and very much aligned with the WHO's prioritized research areas. However, some niche areas of not been researched adequately, as we find from our analysis. For instance, the COVID-19 pandemic's impact is not equal for all; rather, it depends on the demography. Though we have found the shreds of evidence of scientific works that particular focus on the more vulnerable groups like children or pregnant women; however there is a lack of indication regarding the impact analysis on the older adults who are more susceptible to fatality by COVID-19. The analysis also shows the evidence of insufficient studies that focus on the management aspect, such as clinical management, which has a critical role in preventing disease spread and lessening fatality by developing an adequate system and disseminating the information that can offer better health assistance. We have also recognized a small proportion of research contributions by the social science researchers; however, our research cluster analysis or thematic mapping does not reveal any specific information regarding such research. Specially, the WHO emphasizes ethical considerations for research as well as social sciences 
in the outbreak response. Ethical issues have particular importance when several candidate vaccines are in the phase of human trial. Future researchers can take the opportunity to clarify such matters.

Furthermore, from our analysis, we find that one area that is critically lacking sufficient studies is related to 'economics.' Different containment measures taken by the governments worldwide affected economic activities severely, and the world economy has been suffering a lot. However, we fail to detect substantial evidence of research in such an area. It would be more fruitful to analyze economic and policy-related issues in future research. One of the reasons could be the lack of clear guidance regarding the stated area by the WHO in their research roadmap. Perhaps, the regulatory bodies and governments should formulate relevant guidelines to promote scholarly contributions considering our findings. Such initiatives are subject to another aspect of future research - to critically measure the effectiveness of fiscal and monetary stimulus declared by governments from around the Globe. Finally, future studies should focus more on post-pandemic scenarios from different aspects, including public health, governance, and business management.

\section{References}

Aria, M., \& Cuccurullo, C. (2017). Bibliometrix: an R-tool for comprehensive science mapping analysis. Journal of Informetrics, 11(4), 959-975.

Azer, S. A. (2015). The top-cited articles in medical education: a bibliometric analysis. Academic Medicine, 90(8), 1147-1161.

Bieri DS (2019) After the 'Great Half-Century': post-crisis economic geography in retrospect and prospect. Available at SSRN: https://doi.org/10.2139/ssrn.3345700

Bonilla-Aldana, D. K., Quintero-Rada, K., Montoya-Posada, J. P., Ramírez-Ocampo, S., Paniz-Mondolfi, A., Rabaan, A. A., et al. (2020). SARS-CoV, MERS-CoV and now the 2019-novel CoV: have we investigated enough about coronaviruses? - a bibliometric analysis. Travel Medicine and Infectious Disease, 33, 101566.

Campbell, D., Picard-Aitken, M., Côté, G., Caruso, J., Valentim, R., Edmonds, S., et al. (2010). Bibliometrics as a performance measurement tool for research evaluation: the case of research funded by the National Cancer Institute of Canada. American Journal of Evaluation, 31(1), 66-83.

Cobo, M. J., López-Herrera, A. G., Herrera-Viedma, E., \& Herrera, F. (2011). Science mapping software tools: review, analysis, and cooperative study among tools. Journal of the American Society for Information Science and Technology, 62(7), 1382-1402.

Danesh, F., \& Ghavidel, S. (2020). Coronavirus: scientometrics of 50 years of global scientific productions. Iranian Journal of Medical Microbiology, 14(1), 1-16.

Della Corte, V., Del Gaudio, G., Sepe, F., \& Sciarelli, F. (2019). Sustainable tourism in the open innovation realm: a bibliometric analysis. Sustainability, 11(21), 6114.

Demiroz, F., \& Haase, T. W. (2019). The concept of resilience: a bibliometric analysis of the emergency and disaster management literature. Local Government Studies, 45(3), 308-327.

El Mohadab, M., Bouikhalene, B., \& Safi, S. (2020). Bibliometric method for mapping the state of the art of scientific production in Covid-19. Chaos, Solitons \& Fractals, 139, 110052.

Ellegaard, O., \& Wallin, J. A. (2015). The bibliometric analysis of scholarly production: how great is the impact? Scientometrics, 105(3), 1809-1831.

Esfahani, H., Tavasoli, K., \& Jabbarzadeh, A. (2019). Big data and social media: a scientometrics analysis. International Journal of Data and Network Science, 3(3), 145-164.

Feng, Y., Zhu, Q., \& Lai, K.-H. (2017). Corporate social responsibility for supply chain management: a literature review and bibliometric analysis. Journal of Cleaner Production, 158, 296-307. 
Frandsen, T. F., \& Rousseau, R. (2005). Article impact calculated over arbitrary periods. Journal of the American Society for Information Science and Technology, 56(1), 58-62.

Gong, Y., Ma, T.-C., Xu, Y.-Y., Yang, R., Gao, L.-J., Wu, S.-H., et al. (2020). Early research on COVID19: a bibliometric analysis. The Innovation, 1(2), 100027.

Hendrix, D. (2008). An analysis of bibliometric indicators, National Institutes of Health funding, and faculty size at Association of American Medical Colleges medical schools, 1997-2007. Journal of the Medical Library Association: JMLA, 96(4), 324.

Hirsch, J. (2010). An index to quantify an individual's scientific research output that takes into account the effect of multiple coauthorship. Scientometrics, 85(3), 741-754.

Homolak, J., Kodvanj, I., \& Virag, D. (2020). Preliminary analysis of COVID-19 academic information patterns: a call for open science in the times of closed borders. Scientometrics, 124(3), 2687-2701.

Hou, Q., Mao, G., Zhao, L., Du, H., \& Zuo, J. (2015). Mapping the scientific research on life cycle assessment: a bibliometric analysis. The International Journal of Life Cycle Assessment, 20(4), 541-555.

Ji, L., Liu, C., Huang, L., \& Huang, G. (2018). The evolution of resources conservation and recycling over the past 30 years: a bibliometric overview. Resources, Conservation and Recycling, 134, 34-43.

Ki, E.-J., Pasadeos, Y., \& Ertem-Eray, T. (2019). Growth of public relations research networks: a bibliometric analysis. Journal of Public Relations Research, 31(1-2), 5-31.

Liao, H., Tang, M., Luo, L., Li, C., Chiclana, F., \& Zeng, X.-J. (2018). A bibliometric analysis and visualization of medical big data research. Sustainability, 10(1), 166.

Linnenluecke, M. K., Marrone, M., \& Singh, A. K. (2019). Conducting systematic literature reviews and bibliometric analyses. Australian Journal of Management. https://doi.org/10.1177/0312896219 877678

Merigó, J. M., Gil-Lafuente, A. M., \& Yager, R. R. (2015). An overview of fuzzy research with bibliometric indicators. Applied Soft Computing, 27, 420-433.

Nowakowska, J., Sobocińska, J., Lewicki, M., Lemańska, Ż, \& Rzymski, P. (2020). When science goes viral: the research response during three months of the COVID-19 outbreak. Biomedicine \& Pharmacotherapy, 129, 110451.

Salari, N., Hosseinian-Far, A., Jalali, R., Vaisi-Raygani, A., Rasoulpoor, S., Mohammadi, M., et al. (2020). Prevalence of stress, anxiety, depression among the general population during the COVID19 pandemic: a systematic review and meta-analysis. Globalization and Health, 16(1), 1-11.

Secinaro, S., Brescia, V., Calandra, D., \& Biancone, P. (2020). Employing bibliometric analysis to identify suitable business models for electric cars. Journal of Cleaner Production, 264, 121503.

Waltman, L., Calero-Medina, C., Kosten, J., Noyons, E. C., Tijssen, R. J., van Eck, N. J., et al. (2012). The Leiden Ranking 2011/2012: data collection, indicators, and interpretation. Journal of the American Society for Information Science and Technology, 63(12), 2419-2432.

World Health Organization (WHO). (2020). A coordinated global research roadmap: 2019 novel coronavirus. Geneva, Switzerland: The World Health Organization.

Zupic, I., \& Čater, T. (2015). Bibliometric methods in management and organization. Organizational Research Methods, 18(3), 429-472.

Publisher's Note Springer Nature remains neutral with regard to jurisdictional claims in published maps and institutional affiliations.

\title{
Authors and Affiliations
}

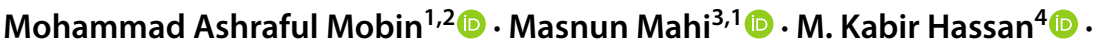 Marzia Habib ${ }^{5}(\mathbb{0})$ Shabiha Akter ${ }^{6,1}[1] \cdot$ Tahmina Hassan $^{7}$}

\author{
Mohammad Ashraful Mobin \\ mobin@ifintell.org \\ Masnun Mahi \\ masnunmahi@um.edu.my; masnunmahi@gmail.com
}


Marzia Habib

marziahabib@hotmail.com

Shabiha Akter

shabihaakter@sd.taylors.edu.my

1 iFINTELL Business Intelligence, Cyberjaya, Malaysia

2 Graduate School of Business, Universiti Sains Malaysia, George Town, Malaysia

3 Faculty of Business and Accountancy, University of Malaya, Kuala Lumpur, Malaysia

4 Department of Economics and Finance, University of New Orleans, New Orleans, LA 70148, USA

5 Medical Analytics Team, iFINTELL Business Intelligence, Cyberjaya, Malaysia

6 School of Accounting and Finance, Taylor's University, Subang Jaya, Malaysia

7 Independent Public Health Researcher, New Orleans, USA 